\title{
Prenatal detection of de novo inversion of chromosome 9 with duplicated heterochromatic region and postnatal follow-up
}

\author{
Jeong Joong Kim ${ }^{1}$, Hee Sub Rhee ${ }^{2,4}$, Yeun Tai \\ Chung ${ }^{1}$, So Yeon Park ${ }^{3}$ and Soo Kyung $\mathrm{Choi}^{3}$ \\ ${ }^{1}$ Department of Anatomy, School of Medicine, Wonkwang \\ University, Iksan 570-749, Korea \\ ${ }^{2}$ Department of Obstetrics and Gynecology, School of Medicine, \\ Wonkwang University, Iksan 570-749, Korea \\ ${ }^{3}$ Genetic Research Laboratory, Samsung Cheil Hospital \& \\ Women's Healthcare Center, Medical Research Institute, \\ Sungkyunkwan University, Seoul 100-380, Korea \\ ${ }^{4}$ Corresponding author: Tel: 0653-850-1239; \\ Fax: 0653-850-6760; E-mail: rh 5710@wonnms.wonkwang.ac.kr
}

Accepted 22 June 1999

Abbreviation: FISH, fluorescence in situ hybridization; PRINS, primed in situ labelling

\begin{abstract}
We report the first de novo case of a heterochromatic duplication on the long arm of the chromosome 9, which then was pericentrically inverted at p11q13. This condition was detected prenatally and carry to term. We then performed the follow up for over 1 year. So far, there seems to be no phenotypical abnormalities.
\end{abstract}

Keywords: heterochromatic duplication, pericentric inversion, de novo inversion

\section{Introduction}

Pericentric inversions of chromosome 9 are among the most frequent chromosomal rearrangements in human, with a frequency of 1-2 percent (Nielson and Silesen, 1975). The majority of these inversions are inherited. Prenatal diagnosis of a de novo inversion poses a dilemma in providing the family with a best possible counselling. In the present case, we characterized the duplication of the classical satellite region and the pericentric inversion of chromosome 9 using $\mathrm{FISH}$ (fluorescence in situ hybridization) in prenatal diagnosis. And we report the physical and developmental follow-up of a patient documented with a de novo inversion of chromosome 9 with duplicated heterochromatic region.

\section{Case Report}

Prenatal cytogenetic diagnosis was performed with amniocentesis in a 28-year-old woman (0-0-0-0) who had Down positive result on the triple test. GTG-banding showed abnormal extension of the long arm on the chromosome 9, which led to a suspicion of translocation of insertion (Figure 1A). To find the origin of the seeming extended region, CBG-banding was performed. We confirmed that the extended region was the duplication of the heterochromatic region (Figure 1B). Futhermore, RBG-banding was followed with the discovery of unstained negative band which confirmed the absence of euchromatic region (Figure $1 \mathrm{C}$ ). In addition, there was no abnormality found by detailed fetal ultrasonography.

Chromosomal analyses on the lymphocytes from both parents were found to be a normal. The parents elected to continue the pregnancy. At 40 weeks, a $3.73 \mathrm{~kg}$ male baby was delivered. Vaginally Chromosome analysis on cord blood taken at birth confirmed the presence of a pericentrically inverted chromosome 9 in all cells examined. The PRINS (primed in situ hybridization) using D9Z1 on interphase and metaphase cells confirmed the chromo-some 9 and using classical alpha-satellite primer, we have found inverted region unobservable by convention-al banding technique (Figure 2). By the FISH method using WCP9, heterochromatic region of pericentrically invert-ed chromosome 9 didn't have observable signal (Figure 3).

Therefore, the duplication of the chromosome 9 of fetus was presumably occurred and then inversion rearrangement of the two break regions took place between the short arm ( $p 11$ ) and the long arm (q13) (Figure 4). On postnatal evaluation, up to now, the baby has been exhibiting a normal phenotypes, both mentally and physically.

\section{Discussion}

The variable amount of heterochromatin present in the secondary constriction region of chromosome 9 (9qh), and its association with pericentric inversions have been the subject of scrutiny and debate (Verma, 1988). 


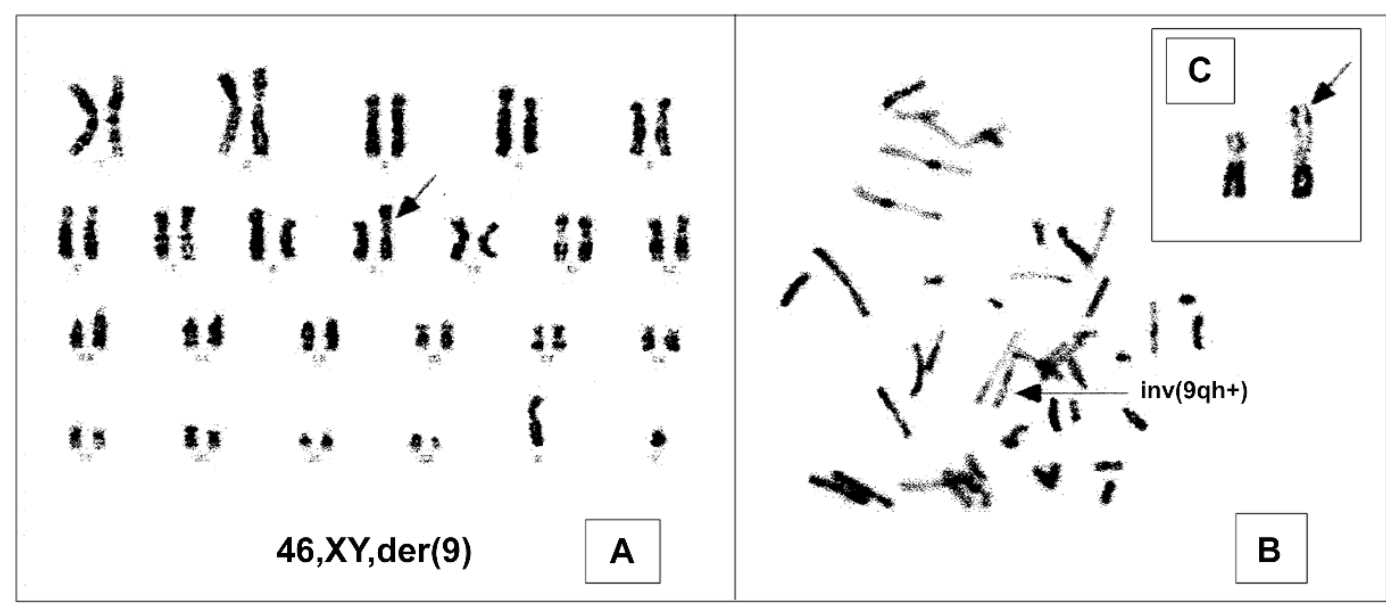

Figure 1. Prenatal diagnosis using GTG-, CBG- and RBG-banding. (A) The arrow indicates the added chromosome by GTG-banding. (B) The region indicated by the arrow is found to be a duplicate of heterochromatin region by CBG-banding. (C) And the arrow shows the absence of dark band (euchromatin negative) on the proximal region of the short arm of chromosome 9 by RBG-banding.

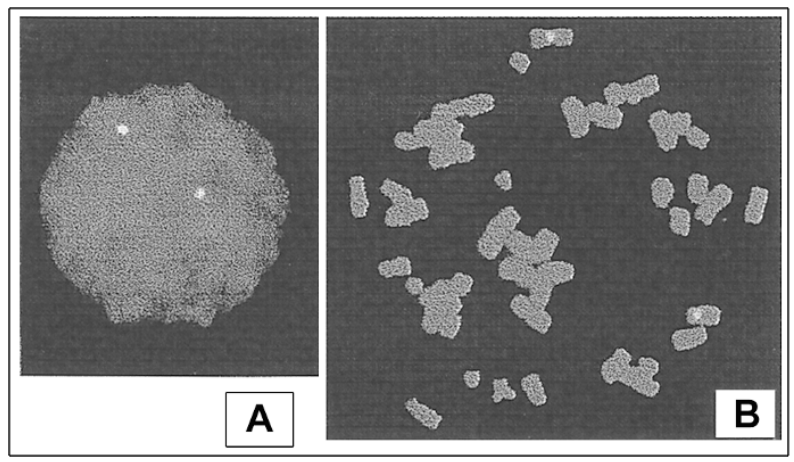

Figure 2. PRINS with specific $\alpha$-satellite DNA primer (D9Z1) for chromosome 9; Both showing two green spots in interphase (A) and metaphase (B).

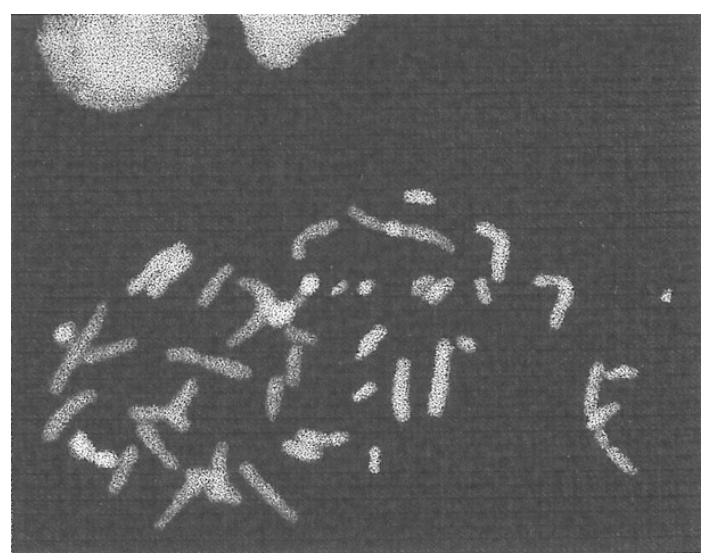

Figure 3. Both chromosomes 9 are painted by fluorescence in situ hybridization (FISH) using whole chromosome painting probe. One shows complete painting but the other incomplete; the unpainted region is the heterochromatin region.

Consequently, attempts have been made to evaluate these regions by various selective techniques. Recent molecular cytogenetic techniques involving FISH have

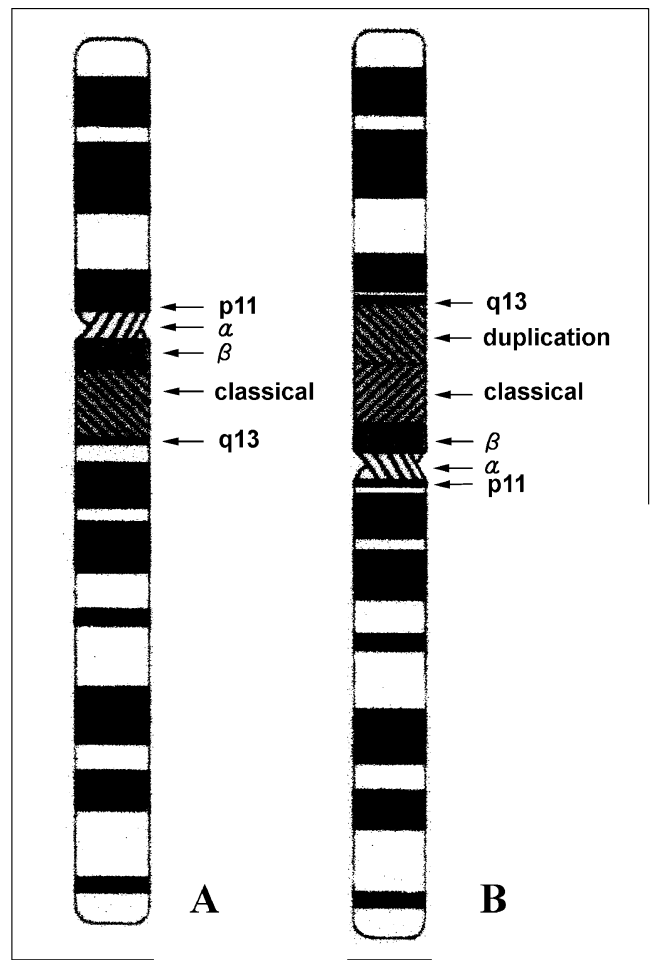

Figure 4. Schematic diagram representing the duplication and the pericentric inversion occurring in chromosome 9 . A is normal chromosome 9 and $B$ is $\operatorname{der}(9) \operatorname{inv}(9)(p 11 q 13) \operatorname{dup}(9)(q 11 q 13)$.

become routine for the characterization of structural variations in the human genome (Bernardi, 1995). Other cytogenetic approaches do not allow in depth characterization and banding techniques reflect the homology at the DNA level (Lohe and Hilliker, 1995). Molecular cytogenetic techniques have unequivocally shown that pericentromeric heterochromatin is not homogeneous in spite of its uniform staining patterns by conventional 
techniques (Singer, 1982). This heterogeneity is presumably due to the arrangement of different fractions of repeated satellite DNA sequences in the pericentromeric heterochromatin (Choo et al., 1991; Tyler-Smith and Willard, 1993). Tandem repeated DNA families have been characterized by FISH analysis using a combination of loci and whole chromosome-specific probes (Adinolfi, 1992).

There are four unique types of pericentric inversions involving the secondary constriction region of chromosome 9 using by fluorescence in situ hybridization technique with human centromere specific $\alpha$ satellite, the $\beta$ satellite (D9Z5) and classical satellite (D9Z1) human DNA probes. Type $A$ inversion consist of breakpoints within the $\alpha$ and $\beta$ satellite DNA regions; type $B$ consist of breakpoints the $\beta$ satellite DNA region 9q13; type $C$ involve breakage within the $\beta$ and classical satellite DNA regions, and type $D$ have breakpoints within the $\alpha$ and classical satellite DNA regions. Like these, obviously, reshuffling of satellite DNA sequences occurred, which has given rise to a variety of heteromorphisms whose clinical significance remains obscure (Samonte et al., 1996), few types of pericentric inversion have been observed due to the variable breakpoints within the qh regions, but their implications have also remained obscure (Verma et al., 1993; Macera et al., 1995).

But, it has been reported that various abnormalities appeared in individuals with the pericentric inversion 9 . In the case of homozygosity for the pericentric inversion 9 , the fetus showed severe intrauterine growth retardation (IUGR), oligohydramnios and subsequently expired in utero (Cotter et al., 1997). Six infertile males with pericentric inversion of chromosome 9 have an abnormality in the hypothalmic-pituitary-testicular axis (Sasagawa et al., 1998). In $2.3 \%$ of the couples with the history of recurrent spontaneous miscarriages, pericentric inversion of chromosome 9 was detected (Sasiadek et al., 1997).

Since chromosomal variation may have an important role in genomic evolution (Houle et al., 1992; Bernardi, 1995) the elucidation of the various heteromorphisms of chromosome 9 constitutive heterochromatin may shed light on this matter. The present study suggests that pericentric inversion have taken place involving the $9 \mathrm{gh}(+)$ region. The formation mechanism involved in the pericentric inversion may be due to the duplication of the classical satellite region (q11q13) of the chromosome 9 and the inversion of the region between the break points (p11 and q13) and its subsequent reunion.

The present case in the first report presenting the $\operatorname{der}(9) \operatorname{inv}(9)(p 11 q 13) \operatorname{dup}(9)(q 11 q 13)$ in Korea as there had been little information on this abnormality in chromosome 9 to make proper interpretation of fetal implication remain obscure. However, as a result of our follow up of this case, we believe that such abnormality alone can not lead to any observable phenotypical abnormalities.

\section{References}

Adinolfi, M. (1992) Identification of chromosome specific satellite DNA using non-isotopic in situ hybridization. In: Verma, R. S. (ed) Advances in genome biology, vol 1. JAI press. Greenwich, Conn. p301-327

Bernardi, G. (1995) The human genome: organization and evolutionary history. Annu. Rev. Genet. 19: 445-476

Choo, K. H., Vissel, B., Nagy, A., Earle, E. and Kalistis, P. (1991) A survey of the genomic distribution of $\alpha$ satellite DNA on all the human chromosome and derivation of a new sonsensus sequences. Nucleic Acid Res. 19: 1179-1182

Cotter, P. D., Babu, A., McCurdy, L. D., Caggana, M., Willner, J. P. and Desnick, R. (1997) Homozygosity for pericentric inversions of chromosome 9 Prenatal diagnosis of two cases. Ann. Genet. 40: 222-6

Houle, D., Hoffmaster, D. K., Assimacopoulos, S. and Charlesworth, B. (1992) The genomic mutation rate for fitness in Drosophila. Nature 359: 58-60

Macera, M. J., Verma, R. S., Conte, R. A., Bialer, M. G. and Klein, V. (1995) Mechanisms of the origin of a G-positive band within the secondary constriction region chromosome 9 . Cytogenet. Cell Genet. 69: 235-239

Nielsen, J. and Sillesen, I. (1975) Incidence of chromosome aberrations among 11,148 newborn children. Hum. Genet. 30: $1-12$

Samonte, R. V., Ramesh, K. H. and Verma, R. S. (1997) Comparative mapping of human alphoid satellite DNA repeat sequences in the great apes. Genetica. 101: 97-104

Sasagawa, I., Ishigooka, M., Kubota, Y., Tomaru, M., Hashimoto, T. and Nakada, T. (1998) Pericentric inversion of chromosome 9 in infertile men. Int. Urol. Nephrol. 30: 203-7

Sasiadek, M., Haus, O., Lukasik-Majchrowska, M., Slezak, R., Paprocka-Borowicz, M., Busza, H., Plewa, R., Bullo, A. and Jagielski J. (1997) Cytogenetic analysis in couples with spontaneous abortions. Ginekol. Pol. 68: 248-52

Singer, M. F. (1982) Highly repeated sequences in mammalian geonme. Int. Rev. Cytol. 76: 76-112

Tyler-Smith and Willard, F. (1993) Mammalian chromosome structure. Cur. Opon. Genet. Dev. 3: 390-397

Verma, R. S. (1988) Heterochromatin: molecular and structural aspects. Cambridge University Press. New York, p276292

Verma, R. S., Luke, S., Brennen, J. P., Mathews, T., Conte, R. A. and Macera, M. J. (1993) Molecular topography of the secondary constriction region(qh) of human chromosome 9 with an unusal euchromatic band. Am. J. Hum. Genet. 52: 981-986 